\title{
Matrix-Based pt router
}

\author{
Kai Nagel
}

\subsection{Basic Information}

\section{Entry point to documentation:}

http://matsim.org/extensions $\rightarrow$ matrixbasedptrouter

\section{Invoking the module:}

http://matsim.org/javadoc $\rightarrow$ matrixbasedptrouter $\rightarrow$ RunMatrixBasedPTRouterExample class

\section{Selected publications:}

Section 3.1 of Nicolai and Nagel (2015); Röder et al. (2013)

\subsection{Summary}

The matrix based PT (Public Transport) router reads a list of PT stops, and constructs "teleported" PT routes using the stops nearest to origin and destination. That is, each resulting trip will approximately look as follows:

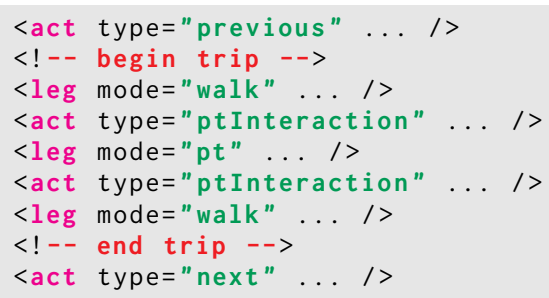

How to cite this book chapter:

Nagel, K. 2016. Matrix-Based pt router. In: Horni, A, Nagel, K and Axhausen, K W. (eds.) The MultiAgent Transport Simulation MATSim, Pp. 133-134. London: Ubiquity Press. DOI: http://dx.doi.org/ 10.5334/baw.20. License: CC-BY 4.0 
The attributes of the walk and the PT legs will be computed from the coordinates of the locations in the same way as teleportation routing (see Section 4.6.2.2), and then taken at face value in the mobsim (see Section 4.6.1.2).

Travel times and travel distances between PT stops can alternatively be given by corresponding matrices. This is particularly useful if a PT assignment exists and such information can be extracted from that. This was used by Röder et al. (2013) and by Zöllig Renner (2014). 